\title{
Understanding nanomedicine size and biological response dependency: What is the relevance of previous relationships established on only DLS- measured Sizes?
}

Elquio Eleamen Oliveira, ${ }^{1,2}$ Maryème Barendji, ${ }^{1}$ Christine Vauthier ${ }^{1 *}$

\footnotetext{
${ }^{1}$ Université Paris-Saclay, CNRS, Institut Galien Paris Sud, 92296 Chatenay-Malabry, France

${ }^{2}$ Centro de Ciências Biológicas e Sociais Aplicadas, Universidade Estadual da Paraíba, João Pessoa, Paraíba, Brazil.
}

Running title: Relevancy of DLS size and biological response dependency?

Published in: Pharm Res 2020 Aug 3;37(8):161. doi: 10.1007/s11095-020-02869-x.

\footnotetext{
*Corresponding author

Christine VAUTHIER

Institut Galien Paris Sud, CNRS UMR 8612,

Univ Paris-Sud, Faculté de Pharmacie,

5 rue J.B. Clément, 92296 Chatenay Malabry (France)

Telephone: + 331468356 03; Fax: + 33146835312

E-mail: christine.vauthier@u-psud.fr
} 


\section{List of Abbreviations}

DLS: Dynamic light scattering

C3A50: Concentration in particles expressed in $\mathrm{cm}^{2} / \mathrm{mL}^{-1}$ required to activate $50 \%$ of the protein $\mathrm{C} 3$ of the complement system in the experimental conditions of the test.

EDTA: Ethylene diamine tetraacetic acid

EGTA: Ethylene glycol tetraacetic acid

IBCA: Isobutylcyanoacrylate

ISO: International Standard Organization

Pdl: Polydispersity Index

PIBCA: poly(isobutylcyanoacrylate)

SDV: Standard deviation

SOP: Standard operating conditions

TEM: transmission electron microscopy

TRPS: Tunable resistive pulse sensing

$\mathrm{VBS}^{2+}$ : Veronal buffer supplemented with calcium $(0.15 \mathrm{mM})$ and magnesium $(0.5 \mathrm{mM})$

VBS5X: Veronal buffer concentrated by 5 times.

VBS-EDTA: Veronal buffer supplemented with EDTA (40 mM)

VBS-EGTA+Mg ${ }^{2+}$ : Veronal buffer supplemented with EGTA (10 mM) and magnesium (2.5 mM) 


\begin{abstract}
Purpose: Most relationships between size and nanomedicine performance and safety were established before the early 2010s' while batch-mode dynamic light scattering (batch-mode DLS) was the only easy size measurement method for colloids available. They are basis for the rational design of nanomedicines, but ununderstood contrasting results are reported. This work aimed to investigate whether these relationships can be used with confidence knowing that batch-mode DLS can be tricky when measuring sizes of polydisperse systems.
\end{abstract}

Methods: A polydisperse dispersion of polymer nanoparticles ranging from 100 to $465 \mathrm{~nm}$ was synthesized. The particles were separated in 4 fractions by successive centrifugations. The capacity of each fraction and parent dispersion to activate the complement system was evaluated by 2D immunoelectrophoresis.

Results: Each fraction was a population of particles with a distinct size. It showed a different capacity to activate the complement system. Particles of the fractions showing the strongest capacity to activate the complement systems had a different size then that of the parent particles.

Conclusion: Particles activating the complement system in the parent dispersion were not those that were detected by batch-mode DLS while measuring its size. This work pointed out that previously established relationships between nanomedicine size and their biological response should be taken with caution if sizes were only measured by batch-mode DLS.

Key words: nanomedicine characterization; dynamic light scattering; particle size distribution; complement system; size-biological response relationship 


\section{Introduction}

The development of nanomedicines has reached a new era with the increased number of formulations receiving approvals by health agencies and the extension of their application in clinic to address unmet medical needs [1-16]. Numerous bottlenecks to their development have been lifted but all difficulties have not yet been resolved [4,16,17-19]. For instance, quality control of nanomedicines remains only partially addressed $[4,16,20-25]$. This procedure, that is applied to demonstrate consistency of nanomedicine performances and safety between batches of synthesis, is based on the measurement of key attributes of the nanomedicine. The small size scale of objects composing nanomedicines complicates the task even considering the evaluation of an obvious attribute such as their size $[16,20,23,25-29]$.

Several methods can be used to evaluate size characteristics of nanomedicines [19, 23-26,29-39]. However, Dynamic Light Scattering applied in the batch mode (batch-mode DLS) remains the most used method $[19,25,27,40,41]$. It is even still the only method considered to characterize the size of nanomedicines in certain works [42,43]. This method was the first for which simple to operate measurement instruments were available favoring their wide implantation in research, industrial and quality control laboratories. The method is recognized to perform nanomedicine size measurements $[24,36,44]$. It was the first for which a standardization by the International standardization organization (ISO) was established $[45,46]$. However, behind easy and automatic measurements, high precautions are needed to prepare samples and to interpret results. The method is very precise and accurate measuring particle size of liquid dispersions composed of spherical particles with a monomodal narrow size distribution using a robust standard operating procedure (SOP) [40,41,47-51]. However, it is unsuitable to measure the size of dispersions having a wide or complex particle size distribution $[19,23,27,28,35]$. This is because the intensity of the scattered light produced by the particles is proportional to the particle diameter raised to the sixth power. Thus, in highly polydisperse dispersions, signals from larger particles can dominate hiding the signal produced by the smallest particles. This effect can generate important bias on size measurements performed on polydisperse dispersions as demonstrated in several works $[19,31,40,52]$. Besides, a low resolution of the method to detect small variations of size distribution was pointed out assessing quality analysis of different batches of Doxil ${ }^{\circledR}$, a liposome-based nanomedicine approved for clinical use since 1995 [28]. Because of these limitations, it is recommended to evaluate the size of nanomaterials including nanomedicines with different methods working with different physic principles [25]. Applying such an orthogonal approach, we have demonstrated that batch-mode DLS missed to report the large and complex size distribution of one of our polymer nanomedicine (different populations in size with $D_{H}$ comprised between 90 to $500 \mathrm{~nm}$ ) providing with a mean hydrodynamic diameter of a single population of particles $\left(D_{H}=220 \mathrm{~nm}\right)$ and a low polydispersity index $(\mathrm{Pdl})(\mathrm{Pdl}=0.08)$. Measurement quality criteria were very good discarding any suspicion of bias on the size measured by batch-mode DLS [52]. We have found this result problematic as the size is one of the key attributes of nanomedicine which means that it influences the biological fate, activity and safety $[9,16,35,53-63]$. It raises questions about relationships between size and nanomedicine efficacy and safety that were established before the years 2010s', at a time DLS was the only size measurement method for colloids available $[54,55,58,59,61,64,65]$. It is noteworthy that these relationships are currently used to draw rational approaches for designing nanomedicines $[9,20,60,63,66-68]$. However, in their critical review of the literature analyzing reports on the influence of the size of nanomedicines on their biodistribution, Gaumet et al. highlighted the occurrence of several inconsistencies between the established relationships [65]. They pointed out the lack of precision on reporting the polydispersity in size of nanomedicines from size measurements performed by batch-mode DLS only as one of possible factors that lead to the contradictory relationships [65]. Obviously, these relationships did not take into account the particle size distribution which recently emerged as another key attribute of a nanomedicine $[9,19,21,25,35,69]$ and can now be better determined using a combination of size measurement methods [See for instance: 52,70-75]. Considering the results of our above-mentioned 
previous work [52], it may also be postulated that our present understanding of the influence of nanomedicine size on biological responses may be based on incorrect sizes as it is impossible to exclude a bias from data given the size only measured by batch-mode DLS. Thus, it is urgent to clarify the relevance of the existence of a possible bias to remove some of the current difficulties in obtaining consistent results when designing nanomedicines [68].

The aim of the present work was to investigate whether the previously established relationships can be used confidently by answering the following two questions

1. Does the size heterogeneity in of the dispersion results in a heterogeneous or homogeneous biological response?

2. Is the observed biological response due to the particles that were detected analyzing the parent dispersion by batch mode DLS or to another size population that this size measurement method missed to detect?

To carry on this work, a dispersion of nanoparticles with a complex size distribution was needed. We have used our previously characterized polymer nanoparticle dispersion [52] that was fractionated in 4 fractions containing different particle populations. The biological response that was chosen to monitor was the capacity of the nanoparticles to activate the complement system that is recommended to evaluate the safety of a nanomedicine $[76,77]$. The activation of the complement system also has determinant role in controlling the in vivo fate of intravenously administered nanomedicines and on their efficacy [78-81] and can be assessed by in vitro tests [82].

\section{Materials and Methods}

\subsection{Materials}

The monomer used for the preparation of the nanoparticles, isobutylcyanoacrylate (IBCA) was purchased from Afinitica (Barcelona, Spain). Nitric acid (Prolabo VWR, Fontenay sous Bois, France), hydrochloride acid (Fluka, Saint-Quentin Fallavier, France), Cerium IV ammonium nitrate (Fluka, SaintQuentin Fallavier, France), dextran 66.7 kDa (Sigma, Saint-Quentin Fallavier, France) were used as supplied.

For the analysis of the activation of the complement system, human serum was prepared from plasma provided by Etablissement Français du Sang (Agreement \# 14/ESF/041, Rungis, France). Anti-human C3 polyclonal antibody raised in goat was purchased from Fitzgerald (Anton, USA). Agarose was supplied by Sigma, Gel-fix ${ }^{\circledR}$ plates for agarose gel $(265 \times 150 \mathrm{~mm})$ were purchased from Serva Electrophoresis (Heidelberg, Germany). Tricine buffer was prepared with tricine at $27 \mathrm{mM}$ (Sigma), Tris Base at $63 \mathrm{mM}$ (Sigma7-g ${ }^{\circledR} \mathrm{c}$, Sigma), Calcium lactate at $1 \mathrm{mM}$ (Fisher Scientific, Illkirch, France). Veronal buffer 5X (VBS5X) was composed of diethylmalonylurea $25 \mathrm{mM}, \mathrm{NaCl} 750 \mathrm{mM}, \mathrm{pH}$ 7.4. Veronal buffer with calcium and magnesium (VBS ${ }^{2+}$ ) was prepared diluting VBS5X by 5 and adding $0.15 \mathrm{mM} \mathrm{CaCl}$ (Sigma) and $0.5 \mathrm{mM} \mathrm{MgCl}_{2}$ (Sigma, Saint-Quentin Fallavier, France). Veronal buffer with $40 \mathrm{mM}$ EDTA (VBS-EDTA) was prepared diluting VBS5X by 5 and adding ethylenediaminetetraacetic acid (EDTA) sodium salt (Sigma, Saint-Quentin Fallavier, France). Veronal buffer with $10 \mathrm{mM}$ ethylene glycol tetraacetic acid (EGTA) and $2.5 \mathrm{mM}$ magnesium (VBS-EGTA+Mg ${ }^{2+}$ ) was prepared diluting VBS5X by 5 and adding EGTA and $\mathrm{MgCl}_{2}$.

\subsection{Methods}

\subsubsection{Preparation of the parent dispersion of nanoparticles}

The parent dispersion of nanoparticles was prepared following the protocol described in Varenne et al. [52]. Briefly, $9.7 \mathrm{~mL}$ of a dextran solution at $51.7 \mathrm{mg} / \mathrm{mL}$ in nitric acid $0.2 \mathrm{M}$ were purged with nitrogen for $10 \mathrm{~min}$ and the temperature was raised to $40^{\circ} \mathrm{C}$. To start the synthesis of the nanoparticles, $0.3 \mathrm{~mL}$ of cerium IV ammonium nitrate $(0.08 \mathrm{M}$ in nitric acid $0.2 \mathrm{M})$ immediately followed by the addition of $0.5 \mathrm{~mL}$ of IBCA were added to the solution of dextran placed under strong 
magnetic stirring. The polymerization was pursued for $50 \mathrm{~min}$ at $40^{\circ} \mathrm{C}$. At the end, the obtained milky dispersion was cooled down rapidly using an ice bath. The dispersion was then dialyzed against milli$\mathrm{Q}^{\circledast}$ water. After dialysis, an aliquot of the dispersion was stored at $+4^{\circ} \mathrm{C}$ until use and the rest was used to prepare fractions of different size

\subsubsection{Preparation of the different fractions in size from the parent dispersion}

Separation of the parent dispersion of nanoparticle in fractions of different size was achieved by successive centrifugations. Figure 1 summarizes the conditions of centrifugation used and the different fractions that were prepared during this fractionation procedure. During the procedure, the weight of each fraction was measured using a balance Mettler Toledo XS105 Dual range to achieve a mass balance of the distribution of the different types of nanoparticles isolated in each fraction.

The different fractions were stored at $+4^{\circ} \mathrm{C}$ until use.

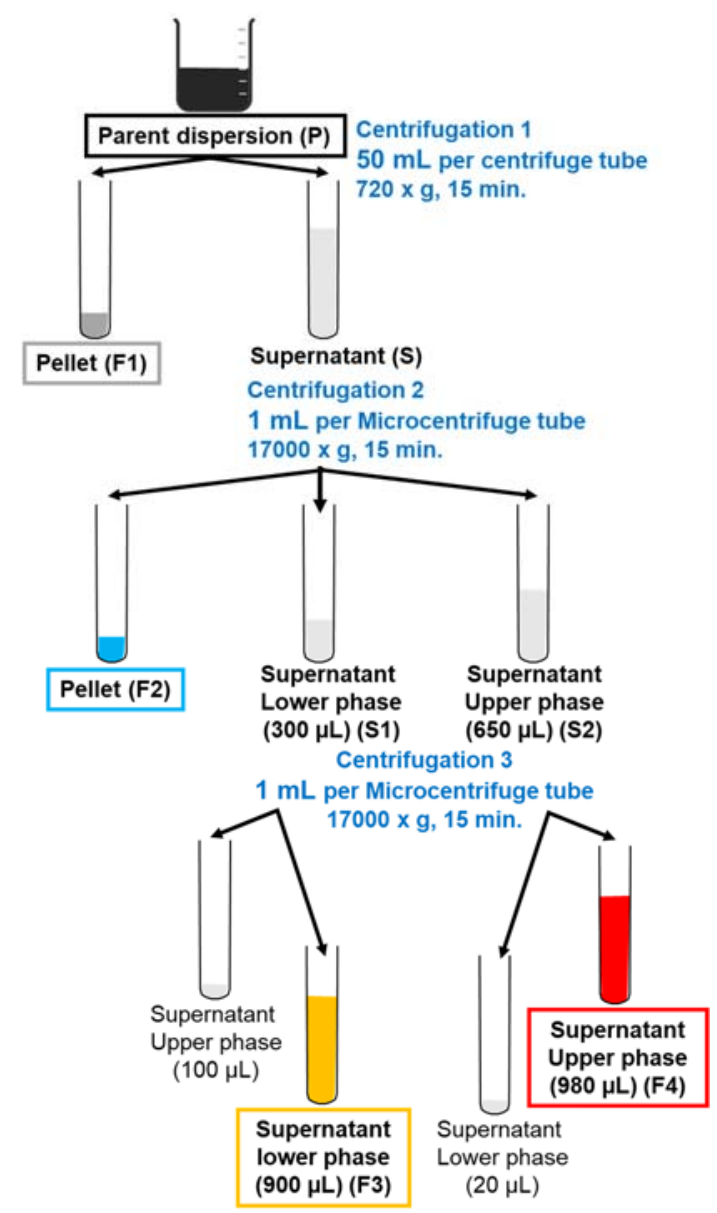

Figure 1: Scheme of experimental conditions used to fractionate the parent dispersion (P). Fractions F1, F2, F3 and F4 kept for further analysis were underlined with the color code used in the different figures. Centrifugation 1 was performed with a Centrifuge 5804R Eppendorf. Centrifugations 2 and 3 were performed using a 5417R Eppendorf centrifuge. Volumes were $50 \mathrm{~mL}$ per tube for centrifugation 1 and $1 \mathrm{~mL}$ distributed in microcentrifuge tubes for centrifugations 2 and 3 .

\subsubsection{Determination of the concentration in particles}

Aliquots $(100 \mu \mathrm{L}$ or $300 \mu \mathrm{L})$ of the parent dispersion and of each fraction were lyophilized for $24 \mathrm{~h}$ in a freeze drier Christ-Alpha 1-2CDPlus (Bioblock Scientific, Illkirch, France). The weight of the dry residue attributed to the nanoparticles contained in the aliquot was determined using a precision balance Mettler Toledo XS105 Dual range. The concentration in particles, $M_{c}$, expressed in $\mathrm{mg} / \mathrm{mL}$ was then 
calculated from the weight of dried residue obtained from the volume of the lyophilized aliquot. The concentration of the dispersion expressed as the area of nanoparticles per volume unit $\left(\mathrm{cm}^{2} / \mathrm{mL}\right)$ was calculated with equation 1

$S=\frac{6 \times M_{c}}{\rho \times D_{H}} \quad$ Equation 1

In which $\rho$ was the volumetric mass of the nanoparticles taking the value of $1.14 \mathrm{~g} / \mathrm{cm}^{3}$ according to Vauthier et al. [83], and $D_{H}$ was the mean hydrodynamic diameter determined by DLS as explained in section 2.2.4.

The number of particles, $N$, was deduced from the mass of particles, $m, D_{H}$ and $\rho$, with equation 2

$N=\frac{6 \times m}{\pi \times \rho \times D_{H}^{3}} \quad$ Equation 2

The application of equation 2 considering $M c$ instead of $m$ gives the concentration of the dispersion expressed in number of particles per $\mathrm{mL}$.

\subsubsection{Characterization of the size and size distribution}

The size and size distribution of the dispersions were evaluated by different methods. The batch-mode DLS which covers a wide range of size was used for all dispersions while other methods were applied depending on their limitations regarding size limits and/or amount of sample needed.

For batch-mode DLS, evaluation of the size of the particles was achieved using a previously validated protocol which applies for the evaluation of the size of polymer nanoparticles $[49,50]$. All cares were used to prepare samples. Measurements were performed using a Zetasizer ZS90 (Malvern Instrument, Malvern Panalytical, Orsay, France) at the optimal concentration determined in previous works for this type of nanoparticles $(0.1 \mathrm{mg} / \mathrm{mL})$ [49] Dilutions were performed with filtered Milli- $\mathrm{Q}^{\circledR}$ water. Prior measurement, the sample was let to equilibrate at the temperature measurement set at $25^{\circ} \mathrm{C}$ for 5 $\mathrm{min}$. Results were expressed as the z-average hydrodynamic diameter and the particle size distribution (PDI) deduced from the cumulant fit.

Size of dispersions $\mathrm{P}, \mathrm{F} 2, \mathrm{~F} 3$ and $\mathrm{F} 4$ were also evaluated at $25^{\circ} \mathrm{C}$ using an analytical photocentrifuge LUMiSizer ${ }^{\circledast} 6514$ (12 measurement channels) (LUM GmbH, Berlin, Germany) and STEP-TechnologyTM [38]. Samples were diluted in Milli- ${ }^{\circledR}$ water to achieve a concentration in nanoparticles ranging from 2 to $10 \mathrm{mg} / \mathrm{mL}$. Volume sample introduced in the measurement cell (Cell LUM $10 \mathrm{~mm}, \mathrm{PC}$, Rect. Synthetic cell (110-132xx)) was $1.3 \mathrm{~mL}$. Centrifugation at a relative centrifugal acceleration of 2300 (at bottom position) were performed for 5 hours. The detector operated at a wavelength of $870 \mathrm{~nm}$ for dispersions P, F2 and F3 and of $410 \mathrm{~nm}$ for the dispersion F4 Results were expressed as the harmonic average value of the diameter deduced from the log normal distribution and standard deviation.

Size of dispersions F2 and F3 were also evaluated by Tunable Resistive Pulse Sensing (TRPS) [37]. The instrument was a Q-Nano (Izon, Lyon, France) operating with nanopore np300 for F2 and np150 for F3. Data acquisition and interpretation was achieved using the IZON Control Suite software version 3.3.2.2001. A calibration of the apparatus was performed using polystyrene calibration particles having a mode size of $115 \mathrm{~nm}$ provided by Thermo Fisher Laboratory and used at a concentration of $1.2 \times 10^{10}$ particles $/ \mathrm{mL}$. Samples were diluted in phosphate saline buffer containing $0.03 \%$ of Tween 20 (Izon solution $Q$ ) at a concentration of $0.05 \mathrm{mg} / \mathrm{mL}$. Results were expressed as the mean diameter in number and standard deviation.

Particles contained in each fraction and in the parent dispersion were observed by transmission electron microscopy (TEM) for qualitative analysis of the shape, size and size distribution. Samples were diluted at a concentration of $0.1 \mathrm{mg} / \mathrm{mL}$ and deposited on formvar carbon coated cupper grids 
for TEM by flotation for $3 \mathrm{~min}$. The excess of sample was let to dry in dust free environment. Grids were then stained for $10 \mathrm{~s}$ with phosphotungstate solution (1\%) by flotation. The excess of staining solution was blotted, and the grid was let to dry before introduction in the electron microscope. Observations were performed using a JEOL JEM1400 electron microscope operating at 80KV at the Electron Microscope facility Imagif (I2BC, CNRS, Gif France). Images were acquired with a Gatan camera ORIUS SC1000A using Digital Micrograph Gatan software.

\subsubsection{Determination of the zeta potential}

Zeta potential of the different dispersions were evaluated using a Zetasizer ZS90 (Malvern Panalytical, Orsay France). The nanoparticles were diluted in a solution of sodium chloride $1 \mathrm{mM}$ filtered thru $0.2 \mu \mathrm{m}$. Operating conditions used to perform measurements were described in Varenne et al. [51]. Results were the mean zeta potential value of 5 measurements performed on 2 independent experiments.

\subsubsection{Evaluation of the activation of the protein $\mathrm{C} 3$ of the complement system by crossed immunoelectrophoresis.}

Complement activation triggered by nanoparticles incubated in human serum was assessed evaluating the cleavage of the protein C3 by 2-D immunoelectrophoresis developed by Passirani et al. [84] and modified by Coty et al. [85] to achieve high throughput testing.

The concentration of nanoparticles that triggered $50 \%$ of the activation of the complement system in our experimental conditions was determined as follow. Test tubes for incubations were prepared in an ice bath with $25 \mu \mathrm{L}$ of $\mathrm{VBS}^{2+}, 50 \mu \mathrm{L}$ nanoparticle sample, $25 \mu \mathrm{L}$ human serum with different concentrations of nanoparticles started from $500 \mathrm{~cm}^{2} / \mathrm{mL}$. The range of concentration in nanoparticles was adjusted for each dispersion to achieve a relevant titration curve for the determination of the activation at $50 \%$ while the upper concentration tested (up to $6000 \mathrm{~cm}^{2} / \mathrm{mL}$ for some dispersions) depended on the initial concentration of the dispersion. Right after addition of the serum that was added last, test tubes were incubated at $37^{\circ} \mathrm{C}$ for 1 hour. At the end of the incubation, test tubes were transferred in an ice bath and $2.5 \mu \mathrm{L}$ of each sample was immediately deposited on the agarose gel to start the $1^{\text {st }}$ dimension of the analysis by crossed immuno-electrophoresis.

In a second set of experiments, incubations were performed with samples prepared with VBSEGTA+Mg ${ }^{2+}$ at the minimal concentration of nanoparticles that produced full activation of the complement system in $\mathrm{VBS}^{2+}$. Samples were incubated at $37^{\circ} \mathrm{C}$ for 1 hour and tested for complement activation as described above.

Few control experiments were added in the different series of experiments to evaluate spontaneous activation of the complement triggered in the experimental conditions and to control serum quality. It is noteworthy that the material used to prepare samples of the control experiments was identical to that used preparing test samples. To evaluate spontaneous activation triggered in the experimental conditions, dispersions of nanoparticles were replaced by water. The quality of the serum was evaluated from two different samples, in one sample, we have added nanoparticles at a concentration that triggered full activation, this sample aimed to verify that the complement system was functional. The second sample aimed to evaluate that the stock serum was not activated during storage. It was prepared with VBS-EDTA $(25 \mu \mathrm{L})$, serum $(25 \mu \mathrm{L})$ and water $(50 \mu \mathrm{L})$. Incubations carried out for the control experiments were performed as described for test samples.

The analysis of the protein C3 of the complement system in incubated samples was achieved by crossed immunoelectrophoresis right after the incubation period. A $1 \%$ agarose gel $(40 \mathrm{~mL}$ in tricine buffer) containing the anti-C3 polyclonal antibody raised on goats was casted on a gel-fix ${ }^{\circledR}$ plate for agarose $(265 \times 150 \mathrm{~mm})$ at $60^{\circ} \mathrm{C}$ as explained in the procedure described by Coty et al. [85] allowing to run 35 analysis at once. At regular intervals, five $0.8 \mathrm{~cm}$ wide bands of gels were removed and replaced 
by plain agarose gel at $1 \%$ prepared in tricine buffer $(1.8 \mathrm{~mL}$ per band). Wells were formed using a specially designed comb forming a raw of 7 holes regularly distributed $(2.5 \mathrm{~cm}$ between each) in the line filled by the plain gel. At the end of the preparation, the gel was placed on the electrophoresis tank (Multiphor II electrophoresis system, GE Healthcare, Buc, France) and loaded with $2.5 \mu \mathrm{L}$ of samples in each well prior to run the first dimension at $600 \mathrm{~V}, 16 \mathrm{~mA}, 100 \mathrm{~W}$. This electrophoresis was stopped when the migration marker incorporated in the gel had migrated by $6.5 \mathrm{~cm}$ (approximately 1h30). To start the second dimension, the gel was rotated by $90^{\circ}$ to achieve the electrophoresis at $500 \mathrm{~V}, 12 \mathrm{~mA}, 100 \mathrm{~W}$ for $3 \mathrm{~h} 30 \mathrm{~min}$. After electrophoresis, the gel was dried for $45 \mathrm{~min}$ with Whatman ${ }^{\circledR}$ paper filters, soak in $0.9 \% \mathrm{NaCl}$ solution for $15 \mathrm{~min}$ to precipitate the proteins, dried for another 30 min with filter papers, soak in water to remove all salts from the gel, and finally dried again with filter paper until complete dryness. Then, the gel was stained with Coomassie blue. Areas under the peaks appearing on each electrophoregrams were measured using image J software. The activation produced by each sample was calculated as the ratio between the area under peaks corresponding to the cleaved protein C3 (proteins of the lower molecular weights) over the total area of peaks shown on the electrophoregram. The value reached 1 when the whole amount of native C3 protein was converted into cleaved fragments indicating a total activation of the protein C3 in the incubated experimental conditions used in this work.

All samples for incubation were prepared a minimum of 3 times from 2 independently prepared dispersions. Each incubated sample was analyzed through the 2D immunoelectrophoresis method a minimum of 3 times. Experimental curves showing the activation of $\mathrm{C} 3$ for the different particle concentrations were fitted with the Boltzmann sigmoid function to determine the concentration of particles activating $50 \%$ of this protein, the C3A50.

\section{Results}

\subsection{Physicochemical characterization of the nanoparticle dispersions}

The parent dispersion was fractionated in different fractions by centrifugation with the aim to separate particles according to their size. The TEM images presented in figure $2 \mathrm{~A}$ shows the heterogeneity in size of particles composing the parent dispersion. Electron micrographs obtained from samples of the fractions highlighted particles with distinct and more define sizes (Figure 2B,C,D,E).

The pellet from the first centrifugation, F1, contained particles of the larger size (Figure 2B). Then the size of the particles found in the different fractions clearly decreased in the order F2, F3 and F4 consistently to what was expected from the application of the fractionation method (Figure 2C,D,E) The larger particles were isolated from pellets in fractions F1 and F2 isolated from the first and second centrifugations. Smallest particles were well isolated in fractions F3 and F4 from the upper and lower phases of the supernatants of the second and third centrifugations.

Size measurements were achieved by batch-mode DLS on all samples. Those performed on the parent dispersion were aimed to identify the population of particles detected by this method. Results were summarized in figure 3 and Table 1. 


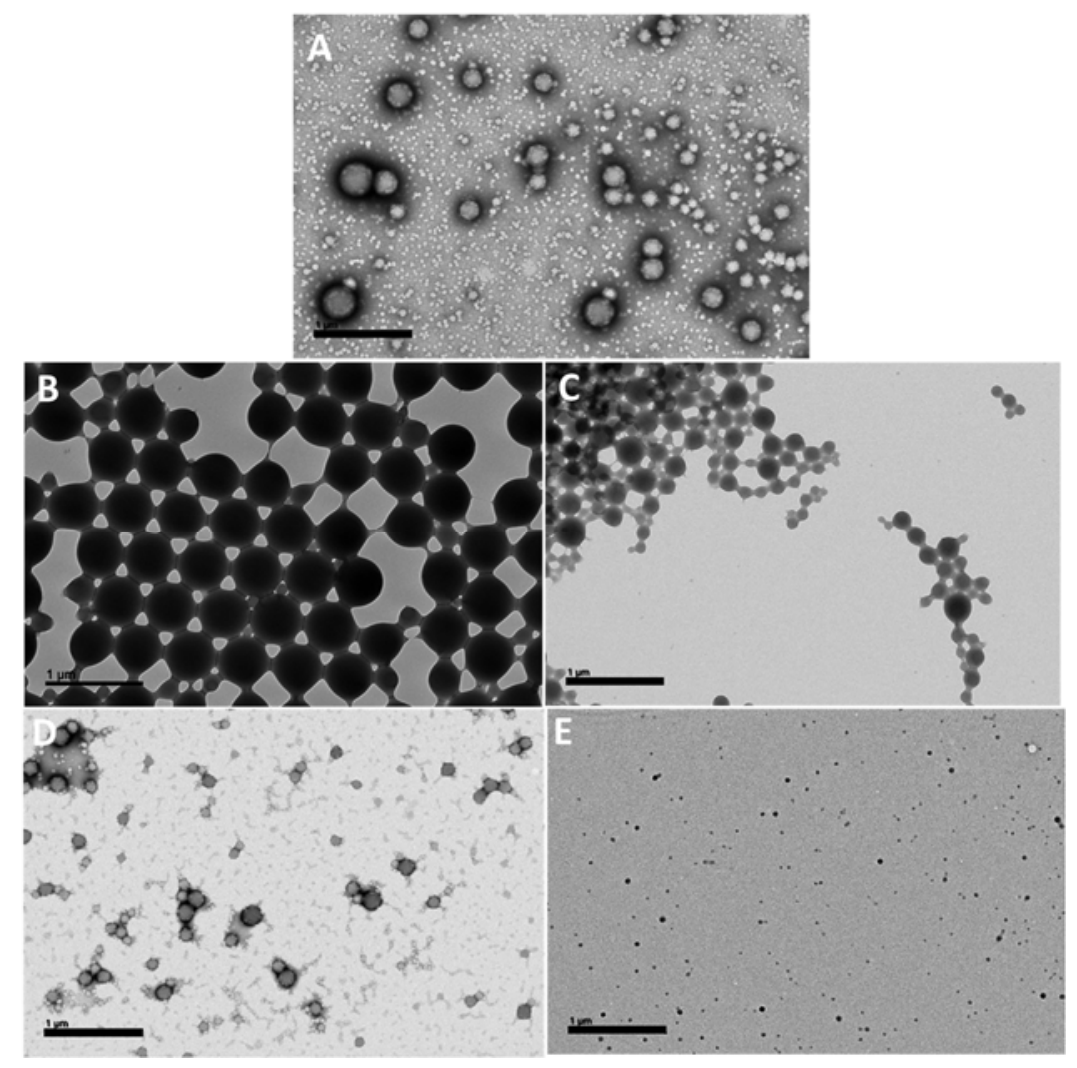

Figure 2: Electron micrographs of the particles composing the parent dispersion $(A)$ and the different fractions isolated from successive centrifugations. B: F1, C: F2, D: F3, E: F4. Scale bar: $1 \mu \mathrm{m}$.

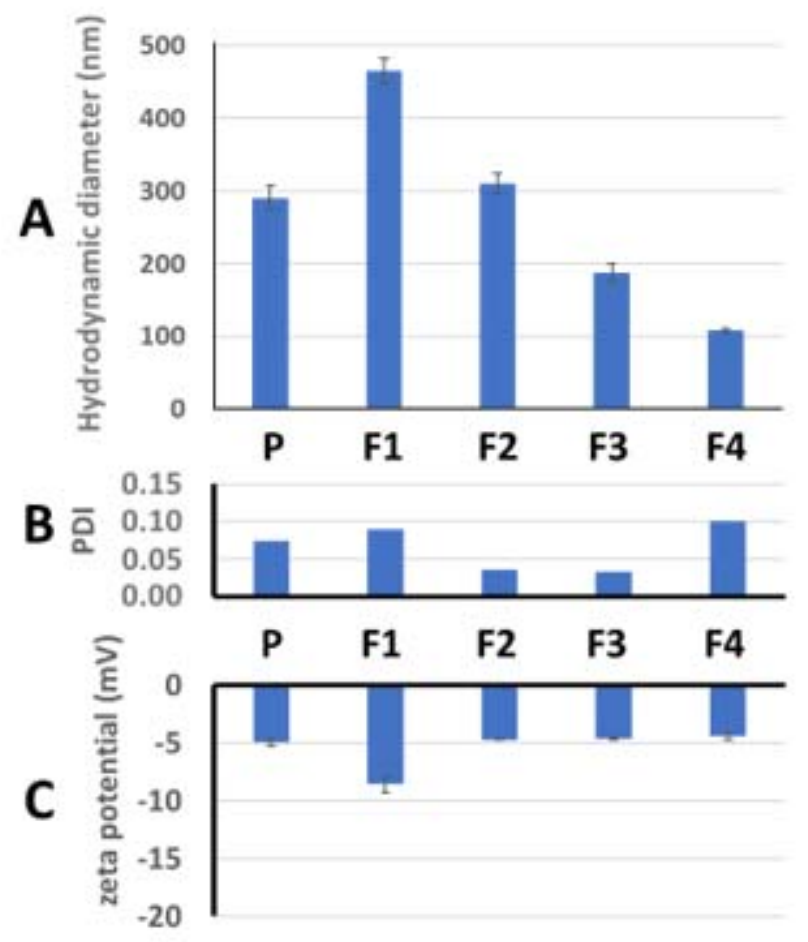

Figure 3: Size and surface charge characteristics of the parent dispersion and of the different fractions as determined by batch-mode DLS and ELS respectively. Hydrodynamic diameter (A), Particle size distribution (B), and zeta potential (C). 
Table 1: Mean diameters of the dispersions evaluated by different methods.

\begin{tabular}{|c|c|c|c|c|c|c|}
\hline & & \multicolumn{5}{|c|}{ Dispersions } \\
\hline Method & Parameter & $P$ & F1 & F2 & F3 & F4 \\
\hline \multirow{3}{*}{ batch-mode DLS } & $\mathrm{D}_{\mathrm{H}}(\mathrm{nm})$ & $290 \pm 16$ & $465 \pm 16$ & $310 \pm 14$ & $187 \pm 13$ & $107 \pm 3$ \\
\hline & width $(\mathrm{nm})$ & 76 & 137 & 54 & 32 & 35 \\
\hline & Pdl & 0.075 & 0.094 & 0.036 & 0.034 & 0.102 \\
\hline \multirow[t]{3}{*}{ TRPS } & $\mathrm{D}(\mathrm{nm})$ & $135^{a}$ & nd & 254 & 120 & nd \\
\hline & Span $^{\text {b }}$ & 0.62 & nd & 0.75 & 0.65 & $\mathrm{Nd}$ \\
\hline & SDV (nm) & nd & nd & 68 & 43 & $\mathrm{Nd}$ \\
\hline \multirow[t]{3}{*}{$\begin{array}{l}\text { Analytical photo } \\
\text { centrifugation }\end{array}$} & $\begin{array}{l}D(\mathrm{~nm}) \\
\text { Log normal }\end{array}$ & 218 & nd & 216 & 110 & 73 \\
\hline & Span $^{\mathrm{b}}$ & 1.4 & nd & 1,34 & 0.73 & 0.97 \\
\hline & SDV & 172 & nd & 162 & 56 & 55 \\
\hline
\end{tabular}

$\mathrm{D}_{\mathrm{H}}$ : hydrodynamic diameter, nd: not determined, SDV: standard deviation, Pdl, polydispersity index. a: from [52], b: span $=\left(D_{90}-D_{10}\right) / D_{50}$

Consistently with TEM observations, the larger particles were found in F1 and diameters of particles found in the other fractions decreased in the order F2, F3, F4. The mean diameter given by DLS for the parent dispersion was very closed to that of the fraction F2 as well as the PDI. The figure 4 shows the size distribution curves obtained for the parent dispersion and fractions as determined by different size measurement methods. Each fraction showed a distinct peak indicating that different populations in size were isolated by our method of fractionation. Consistently with previous works, the distribution curve obtained by batch-mode DLS for the parent dispersion showed a single narrow peak (figure $4 \mathrm{~A}$ black curve). This curve superimposed quite well with that obtained from the analysis of the fraction F2 (figure 4A blue curve). Size distribution evaluated by the analytical photocentrifugation method also showed different peaks for the 3 fractions that could be analyzed by this technique. Position of peaks were consistent with results obtained by batch-mode DLS (Figure 4A, B). The distribution curve of the parent dispersion was almost superimposed with that of $F 2$, but caution should be taken with this result. The size distribution of the parent dispersion, $\mathrm{F} 2$ and F3 were evaluated at the same wavelength of $870 \mathrm{~nm}$ while results shown for F4 were acquired at a different wavelength $(410 \mathrm{~nm})$. The use of a lower wavelength performing size measurement on F4 was required to improve the quality of detection.

When enough sample was available, size of the fractions was also determined by TRPS (Figure 4C). Results showed the same trend compared with those obtained by batch-mode DLS and analytical photocentrifugation (Figure 4A, B). Peaks given by each fraction appeared at a different position indicating that they contained particles of a distinct size. This technique also allows a direct measurement of the concentration of dispersions expressed in number of particles per volume unit. Concentrations measured for $\mathrm{F} 2$ and $\mathrm{F} 3$ were $4.30 \times 10^{12}$ and $7.87 \times 10^{12}$ particles per $\mathrm{mL}$, respectively. These values were of the same order of magnitude than those calculated from the concentration of the dispersions in mass $\left(139.0 \mathrm{mg} / \mathrm{mL}\right.$ and $25.7 \mathrm{mg} / \mathrm{mL}$ for $\mathrm{F} 2$ and $\mathrm{F} 3$, respectively), the mean $D_{H}$ of the particles determined by DLS (table 1 ) and the volumetric mass of the particles [83] which gave $7.82 \mathrm{x}$ $10^{12}$ particles per $\mathrm{mL}$ for $\mathrm{F} 2$ and $6.58 \times 10^{12}$ particles per $\mathrm{mL}$ for $\mathrm{F} 3$.

Particle mean diameters determined by the different methods were summarized in the table 1 . The expression of the diameter between methods was not homogenous hampering a direct comparison of the absolute values $[40,48]$. Apart from this limitation, results appeared consistent between methods, provided that largest and smallest particles were found in the same fractions. 


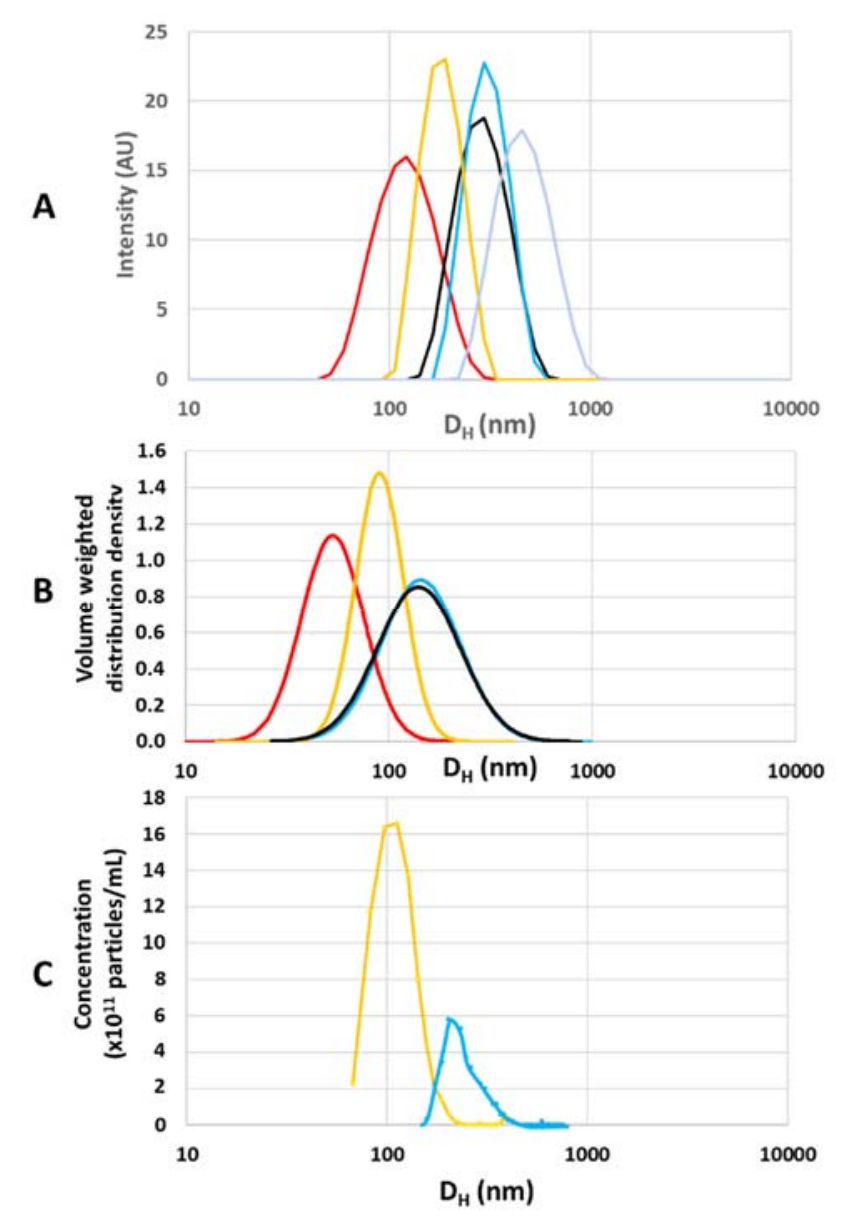

Figure 4 Size distribution curves obtained by A: batch-mode DLS, B: Analytical photocentrifugation (detection wavelength at 870 for the parent dispersion (P), F2, F3 and at $410 \mathrm{~nm}$ for F3), C: TRPS. Black curve: parent dispersion, Grey curve: F1, blue curve: F2, orange curve: F3, red curve: F4.

The zeta potential of the different dispersions was evaluated by electrophoresis light scattering. All dispersions showed a slightly negative value $(\zeta \approx-5 \mathrm{mV}$ ) (Figure $3 \mathrm{C}$ ). Differences between samples were included within the precision of the method. It can be concluded that the zeta potential of the different populations of particles composing the parent dispersion was homogenous. The zeta potential found for the parent dispersion $-5.0 \pm 0.3 \mathrm{mV}$ was consistent with those found in previous works for this dispersion [86-88].

The figure 5 gives the percentage in mass, volume, surface and number of particles isolated in the different fractions composing the parent dispersion. Particles of the fraction $\mathrm{F} 2$ that were detected by batch-mode DLS during size measurement of the parent dispersion represented $62 \pm 2 \%$ in mass and volume of the total amount of particles but only $13.4 \pm 2 \%$ in number. The percentage in surface of the different fractions was provided to give an indication of the area developed by corresponding populations of nanoparticles in the parent dispersion. This information was useful to interpret results from the evaluation of the capacity of the different populations of particles to trigger the activation of the complement system. It is noteworthy that half ( $51 \pm 2 \%)$ of the surface developed by the particles in the parent dispersion was due to the smallest particles isolated in F3 and F4. The other half $(49 \pm 2$ $\%)$ of the surface was due to the larger particles of F1 and F2. The corresponding percentages in mass or volume were $32 \pm 2 \%$ and $68 \pm 2 \%$ respectively. In number, particles with the smallest size (F3 and F4) represented $73.0 \pm 5 \%$ of the total number of particles composing the parent dispersion while those of the larger size isolated in F1 represented only $0.40 \pm 0.04 \%$. 


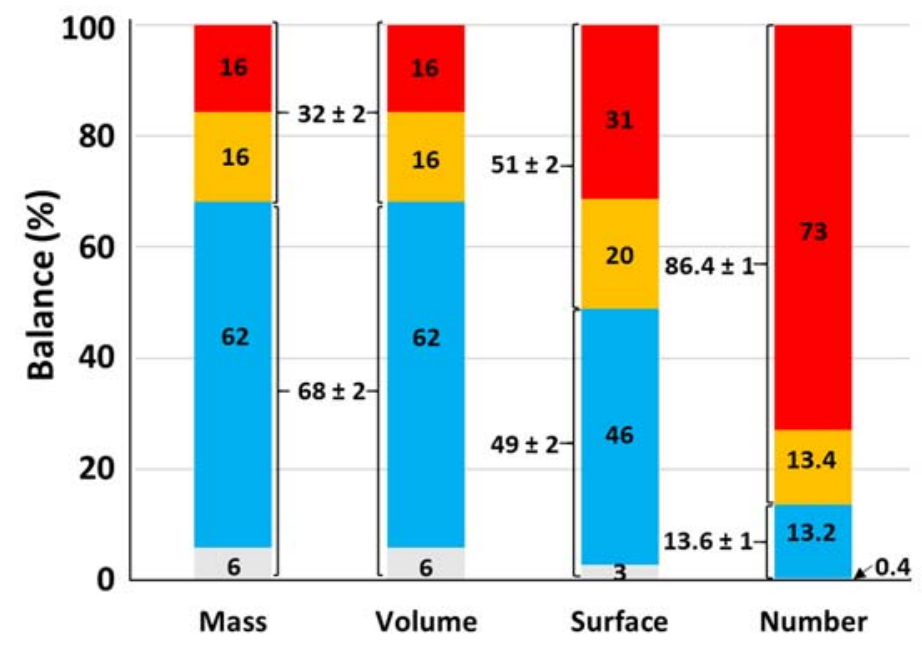

Figure 5 Balance of the different particle populations composing the parent dispersion expressed in mass, volume, surface and number. From bottom to top: light grey: F1, blue: F2, orange, F3, red: F4.

\subsection{Evaluation of the capacity of the particles to activate the complement system.}

A study of the activation of the complement system was performed at different concentrations of particles expressed in surface developed by particles per volume unit to determine the concentration activating $50 \%$ of the protein C3 (C3A50). Curves obtained from the analysis of the parent dispersion and fractions F2, F3 and F4 showed a S-shape which could be fitted with the sigmoidal Boltzmann function (Figure 6). The C3A50 indicated on the figures were deduced from the fit of the curves. With fraction $\mathrm{F} 1$, the activation of the protein $\mathrm{C} 3$ remained below $20 \%$ even at the highest concentration of particles that could be tested (up to $5000 \mathrm{~cm}^{2} \cdot \mathrm{mL}^{-1}$ ). The C3A50 was extrapolated from the fit of the curve with the sigmoidal Boltzmann function established from the experimental points of the first part of the sigmoid. The C3A50 can be used to compare the capacity of different particle populations to activate the complement system. The C3A50 obtained from evaluations performed on the parent dispersion $\left(1650 \mathrm{~cm}^{2} \cdot \mathrm{mL}^{-1}\right)$ and the fractions $\mathrm{F} 3$ and $\mathrm{F} 4\left(1180 \mathrm{~cm}^{2} \cdot \mathrm{mL}^{-1}\right.$ and $1140 \mathrm{~cm}^{2} \cdot \mathrm{mL}^{-1}$ respectively) were of the same order of magnitude. The C3A50 for the fraction $\mathrm{F} 2$ was found at $3000 \mathrm{~cm}^{2} \cdot \mathrm{mL}^{-1}$ while that of the fraction $\mathrm{F} 1$ was extrapolated at $9000 \mathrm{~cm}^{2} \cdot \mathrm{mL}^{-1}$. The graph of the figure 7 reports the value of the C3A50 determine for each fraction as the function of their size. It also includes data recorded for the parent dispersion. Experimental results reported on this graph represent mean values and standard deviations of data collected from several series of completely independent experiments including the preparation of the fractions from different synthesis of parent dispersions. This may explain the quite large error bar on the $Y$ axis for the point plotted at a mean diameter of $310 \mathrm{~nm}$.

It seemed that the variation of C3A50 in this region of size was high. For instance, at the two extreme values of diameters obtained for the fraction F2 isolated from different parent dispersions that are within the precision of the method $(297 \pm 3 \mathrm{~nm}$ and $323 \pm 4 \mathrm{~nm}), C 3 A 50$ varied a lot from $3700 \mathrm{~cm}^{2} . \mathrm{mL}^{-}$ ${ }^{1}$ and $6000 \mathrm{~cm}^{2}$. $\mathrm{mL}^{-1}$ respectively. It is noteworthy that the C3A50 evaluated for the parent dispersion was at the same level than that found for fractions F3 and F4 but there is a bias in the size as pointed out before. 

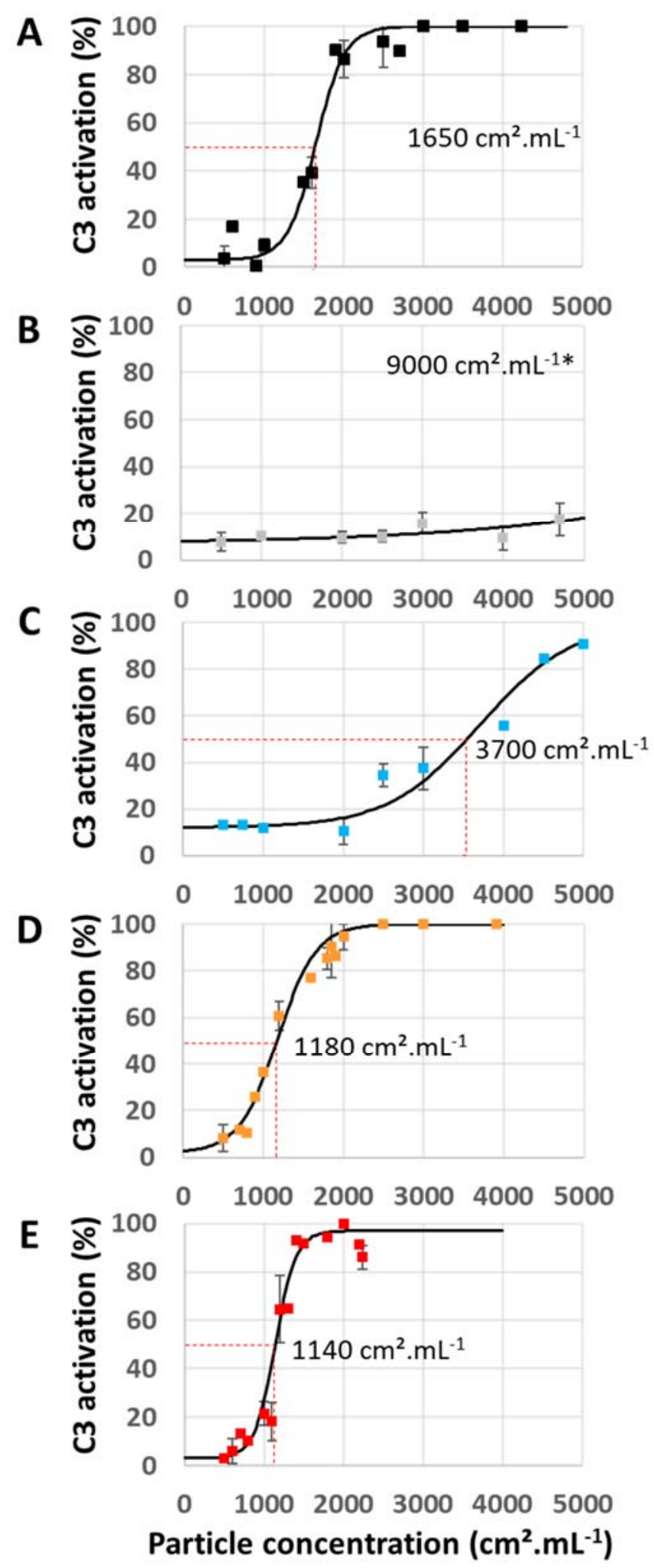

Figure 6: Activation of the $\mathrm{C} 3$ protein evaluated at different concentrations of particles. Curves were fitted with the sigmoidal Boltzmann function (full line), Numbers indicated on graphs are C3A50 deduced from the fit. The value for the fraction F1 (B) was extrapolated from the fit established from the experimental points. A: parent dispersion, B: fraction F1, C: fraction F2, D: fraction F3, E fraction F4. 


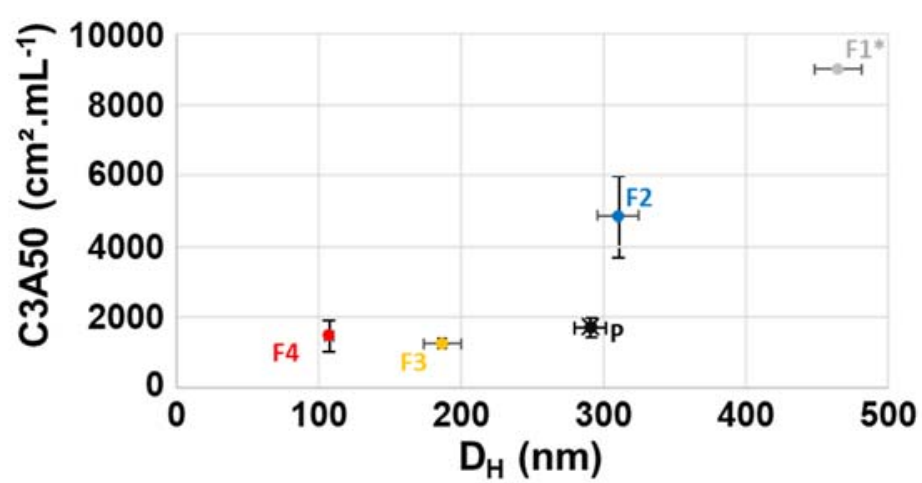

Figure 7: Relation between the size of the particle population of the fractions (F1, F2, F3, F4) and of the parent dispersion (P) and the capacity to activate the complement system expressed as the C3A50. * value extrapolated from the fit of the first part of the curve with the Boltzmann function.

All previous results were obtained while the serum was exposed to nanoparticles in conditions where the activation of the complement system can be triggered by the different pathways. Among those, the classical and lectin-binding protein pathways are calcium dependent. In contrast, the alternative pathway requires only the presence of magnesium. Performing experiments in the absence of calcium in samples can be used to reveal the part of the activation monitored in the previous conditions that was due to the activation by the calcium dependent pathway including the alternative route [89-91]. Figure 8 compares results of complement activation given by our dispersions after incubation in a calcium depleted media (VBS-EGTA+ $\mathrm{Mg}^{2+}$ ) and in $\mathrm{VBS}^{2+}$ containing both calcium and magnesium. This experiment was performed at a concentration of particles that produced a full activation of the complement system in $\mathrm{VBS}^{2+}$ explaining why all dispersions were not tested at the same concentration. The fraction F1 could not be included in this study because its capacity to activate the complement system in VBS ${ }^{2+}$ was low even at a concentration of particles of $5000 \mathrm{~cm}^{2} \cdot \mathrm{mL}^{-1}$ that was the highest concentration that would have been tested with this fraction (Figure 6B). When incubations were performed in the calcium depleted media (VBS-EGTA $\mathrm{Mg}^{2+}$ ) the activation of the protein C3 was markedly reduced but not annealed. The same amplitude of reduction was observed for the different dispersions suggesting that the mechanism of activation of the complement system was homogenous between the parent dispersion and the fractions hence throughout the different populations of particles composing the parent dispersion. The reduction of activation observed in the absence of calcium acknowledged that particles partly activated the complement system by calcium dependent pathways (the classical pathway, the lectin binding protein pathway). Because activation was not completely annealed in the absence of calcium, activation was also produced by the calcium independent route including the alternative pathway.

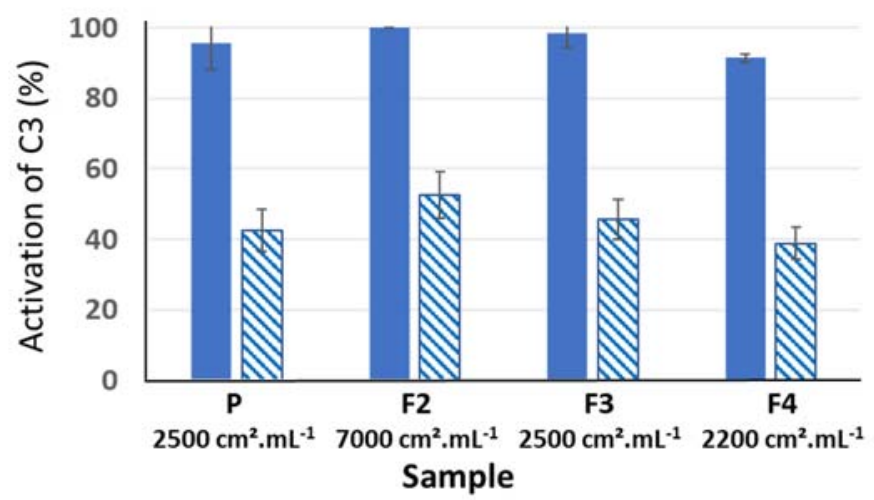

Figure 8: Activation of the complement system triggered through all pathways (full bars: $\mathrm{VBS}^{2+}$ ) and through calcium independent pathway only (hatched bars: VBS-EGTA $+\mathrm{Mg}^{2+}$ ). 


\section{Discussion}

Relationships between size of nanomedicines on their biological response are currently used to build rational basis for nanomedicine formulations. However, they were mostly established with sizes measured by batch-mode DLS that was the sole size measurement method available at this time $[54,55,58,59,61]$. Because this method can generate bias on size determination $[19,25,28,31,40,52]$, it cannot be excluded that such relationships might be questionable. This work was aimed to evaluate this risk investigating the biological response of different populations of particles isolated from a parent dispersion for which batch-mode DLS provided with a biased evaluation of the size as demonstrated in our previous work [52]. The first and second parts of this discussion were aimed to characterize the size and biological response of the different populations isolated from the parent dispersion and to discuss their property regarding that of the parent dispersion. The last part analyzed how the bias in the parent dispersion size measured by batch-mode DLS can affect our understanding of the relationship between size and biological response of nanomedicines.

\subsection{Characterization of the size of the different fractions and of the parent dispersion}

The method of fractionation applied on the parent dispersion produced 4 fractions of particles of distinct sizes. Size measured by different techniques provided with consistent results although the absolute values differed slightly as the expression of the diameter was not homogenous between methods. The population of particles detected by batch-mode DLS in the parent dispersion could be identified. It mainly included the particles isolated in the fraction F2. Maps giving a quantitative representation of each fraction in the parent dispersion could be established in percentage of mass, volume, surface and particle number (Figure 5). From the data given in particle number and the size of the corresponding particles, a theoretical contribution to the intensity of light scattered, l, by each fraction in the parent dispersion can be calculated providing that it is proportional to the number of scattered particles, $n$, and to the power sixth of the particle size, $D_{H}$, as indicated in the equation 3 .

\section{$I \propto n \times D_{H}^{6} \quad$ Equation 3}

Results of this calculation explained why the batch-mode DLS method was unable to decrypt the wide size distribution of our parent dispersion (Figure 9A). The intensity of the scattered light given by particles of the fraction F2 represented $73.5 \%$ of the signal, that of the particles of the fraction F1 participated for $22.2 \%$ of the intensity of the scattered light of the parent dispersion making the contribution of the larger particles to $95.7 \%$ of the total intensity of the scattered light monitored during analysis of the parent dispersion. Participating for only 3.6 and $0.7 \%$ respectively of the intensity of the light scattered by the parent dispersion, fractions F3 and F4 were ignored by batch-mode DLS during measurement of the parent dispersion size. By multiplying the percentages of the scattered light contribution of each fraction by an arbitrary factor of 0.3 , the points of the calculated theoretical contributions to the intensity for each fraction matched with the experimental curve giving the intensity of the scattered light vs particle diameter obtained from batch-mode DLS analysis of the parent dispersion (Figure 9B). This is a new experimentally based evidence of known limits of the batch-mode DLS method to report consistent size measurements for dispersions composed of different populations of particles which size difference is low (below 3 to 5 folds) $[25,39]$. It stressed the importance to confront size measurements given by this method with those obtained from other methods performing orthogonal sizing determinations with techniques based on different principles. This result also pointed out that we need to take with caution size characteristics of dispersions of nanomaterials including nanomedicines that were only determined by batch-mode DLS. 

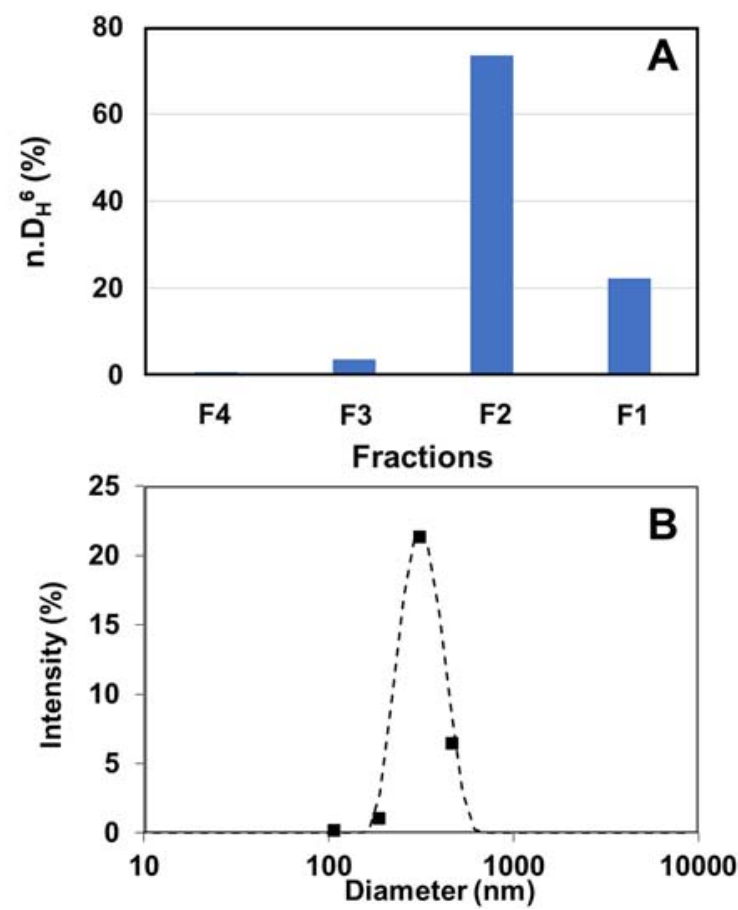

Figure 9: Theoretical evaluation of the contribution of each fraction to the scattered light produced by the parent dispersion. A: Calculation of the contribution to the scattered light produced by different isolated fractions in the parent dispersion. Data were presented with the fraction containing particles of the lower size (F4) on the left and that containing particles of the larger size (F1) on the right. B: Comparison between the experimental curve showing the intensity of the scattered light obtained during batch-mode DLS analysis of the parent particles and of the theoretical contribution calculated for each fraction of particles.

The size characterization of the parent dispersion and fractions achieved by analytical photocentrifugation led to a similar conclusion then that obtained by batch-mode DLS. Indeed, the size distribution shown by the parent dispersion followed that of the fraction F2 (Figure 4B). This observation should be taken with caution as all measurements were not performed using consistent conditions of detection. A lower wavelength of detection $(410 \mathrm{~nm})$ was used to measure the size of the smallest particles isolated in the fraction F4. It might be that the optical system of the analytical centrifuge was unable to detect these small particles at $870 \mathrm{~nm}$, the wavelength used to measure the size of the parent dispersion and of the other measured fractions. It would be worth to clarify this effect in a work dedicated to the evaluation of the performance of this method for which instruments were recently introduced on the market [38].

\subsection{Capacity of the different fractions to activate the complement system}

Key attributes of nanomedicines include the particle size being reported to influence their biological fates $[9,16,35,54,58,60-63]$. Studies on the influence of the size of nanomedicine on the response given by a biological system were generally achieved considering independent formulations of nanomedicine $[53,55,57-59,64,65]$. This is in contrast with the present work in which populations of nanoparticles of different sizes were isolated from a single parent dispersion. This made possible the evaluation of the response of a biological system within size variation of particles composing a single dispersion. The biological response studied in the present work was the capacity of the particles to activate the complement system determining the concentration of particles activating $50 \%$ of the protein $\mathrm{C} 3$ of the complement system, the C3A50, and calcium dependence/independence of the activation pathways. The different fractions of particles showed different capacity to activate the protein C3 of the complement system while the activating particles all triggered activation through similar pathways. In 
our dispersion, the smallest particles showed the highest capacity to activate the protein C3. Results were consistent with that expected from the fact that the particle size can influence the biological response triggered by particles. However, they point out that a single dispersion may contain particles having very different capacities to trigger a given biological response. This may have consequences on the biological performances and safety of the parent dispersion of nanomedicines as the different population of particles of the dispersion will not behave consistently.

4.3. Fractions of particles responsible for the biological response monitored in the parent dispersion

The capacity of the parent dispersion to activate the complement system was at the same level than that of the particles isolated in the fractions F3 and F4 (Figures 6,7). Thus, the biological response monitored while testing the capacity to activate the complement system of the parent dispersion can be attributed to the contribution of the particles of the smallest sizes. These populations represented the minority of the composition in particles of the parent dispersion expressed in mass and volume (32 $\pm 2 \%$ for both). In contrast, they represented the larger population of particles in number $(86.4 \pm 1 \%)$ and their surface represented about the half $(51 \pm 2 \%)$ of the total amount of surface developed by the particles in the parent dispersion. This points out that a parent dispersion of particles can include populations having very different biological activity according to its particle size distribution. A particle population even represented at a low concentration in mass can trigger the strongest biological response while a population which concentration in mass is the higher can remain silencing regarding a given biological response.

It is noteworthy that populations of particles that produced the strongest biological response were those that were not detected by batch-mode DLS characterizing the size of the parent dispersion. This appears to us to be a major problem for the understanding of the role of the size of nanomedicines in their biological performance and safety. Before having made the present work, we would have attributed the strong capacity of the parent dispersion to activate the complement system monitored to particles having a diameter of $290 \pm 16 \mathrm{~nm}$. In the light of the present results, this conclusion is incorrect and suggests that previously established relationship between nanomedicine size determined by batch-mode DLS only and biological response should be taken with caution. It may explain why contrasting results were reported in recent works while attempting to formulate nanomedicines designed on rational basis using these relationships. Because of the conclusion drawn from the present analysis of the data, the fact that the smallest particles produced the strongest activation of the complement system contrasting with the expected results based on previously established size-biological responses relationships will not be further discussed.

The study monitored the capacity of the particles to activate the complement system. Such a biological response was reported to cause serious safety issues in patients treated with nanomedicines $[76,77]$. The complement system also has a role defining the in vivo fate of nanomedicine administered by the intravenous route [78-81]. Results from this work stressed the urgent need to carefully evaluate the particle size distribution of a dispersion of nanomedicines using relevant measurement methods to anticipate any possible heterogeneity of the biological response between different populations of particles composing a single formulation.

\section{Conclusion}

One of the key attributes of nanomedicine is their size characteristics. DLS was for long the only method available to characterize the size of nanomedicine and currently remains the only size measurement method used in several works. Results from this work highlighted that the heterogeneity in size of a dispersion can generate heterogenous biological response consistently with the fact that biological response can be influenced by nanomedicine size. The most important result of this work showed that the population of particles detected by DLS in the parent dispersion was not the 
population which produced the strongest biological activity based on the evaluation of the capacity of the particles to activate the complement system. This result was disturbing suggesting that previous relationships between nanomedicine size and biological responses are questionable being established on size measurements made by DLS only. It emphasizes the need to strengthen nanomedicine size characterization using methods of high resolution that will confidently decrypt size heterogeneity and provide with a more relevant evaluation of the particle size distribution. It supports the urgent need to improve nanomedicine size measurement practices to assess their quality for clinical use and to provide with reliable understanding of their activity and safety according to their size characteristics.

\section{Acknowledgements}

This study was financed in part by the Conselho Nacional de Desenvolvimento Científico e Tecnológico (CNPq) with a fellowship for E.E.O. - Finance Code 245564/2012-8. The authors would like to thank the kind participation of Camille Roesch, Q-Nano, Izon, Lyon and Sylvain Gressier from Lum Gmbh, Berlin for having perform size measurements by TRPS and analytical photocentifugation respectively. The present work has benefited from the electron microscopy platform of the Imagerie-Gif core facility supported by l'Agence Nationale de la Recherche (ANR-11-EQPX-0029/Morphoscope, ANR-10-INBS04/FranceBiolmaging; ANR-11-IDEX-0003-02/ Saclay Plant Sciences).

\section{References}

1 Wicki A, Witzigmann D, Balasubramanian V, Huwyler J. Nanomedicine in cancer therapy: challenges, opportunities, and clinical applications. J Control Release. 2015;200:138-157.

2 Juliano R. The delivery of therapeutic oligonucleotides. Nucleic Acids Res. 2016;44:6518-6548.

3 Mura S, Couvreur P, Combining imaging and drug delivery for the treatment of severe diseases. In: Mura S, Couvreur P, editors. Nanotheranostics for personalized medicine. Singapore: World Scientific Publishing Co. Pte. Ltd. 2016. p. 1-6.

4 Hare JI, Lammers T, Ashford MB, Puri S, Storm G, Barry ST. Challenges and strategies in anti-cancer nanomedicine development: An industry perspective. Adv Drug Deliv Rev. 2017;108:25-38.

5 Lacombe S, Porcel E, Scifoni E. Particle therapy and nanomedicine: state of art and research perspectives. Cancer Nanotechnol. 2017;8:9.

6 Luque-Michel E, Imbuluzqueta E, Sebastián V, et al. Clinical advances of nanocarrier-based cancer therapy and diagnostics. Expert Opin Drug Deliv. 2017;14:75-92.

7 Pelaz B., C. Alexiou, R. Alvarez-Puebla, et al. Diverse Applications of Nanomedicine. ACS Nano. 2017;11:2313-2381.

8 Ventola CL. Progress in Nanomedicine: Approved and Investigational Nanodrugs. P T. 2017;42:742-755.

9 Cicha I, Chauvierre C, Texier I, Cabella C, Metselaar JM, Szebeni J, et al. From design to the clinic: practical guidelines for translating cardiovascular nanomedicine. Cardiovasc Res. 2018;114:1714-1727.

10 Dormont F, Varna M, Couvreur P. Nanoplumbers: biomaterials to fight cardiovascular diseases. Materials Today 2018;22:122-143.

11 Dormont F, Rouquette M, Mahatsekake C, Gobeaux F, Peramo A, Brusini R, et al. Translation of nanomedicines from lab to industrial scale synthesis: The case of squalene-adenosine nanoparticles. J Control Release. 2019;307:302-314.

12 Rodríguez-Nogales C, González-Fernández Y, Aldaz A, Couvreur P, Blanco-Prieto MJ. Nanomedicines for Pediatric Cancers. ACS Nano. 2018;12:7482-7496.

13 Valero L, Alhareth K, Gil S, Lecarpentier E, Tsatsaris V, Mignet N, et al. Nanomedicine as a potential approach to empower the new strategies for the treatment of preeclampsia. Drug Discov Today. 2018;23:1099-1107.

14 Couvreur P. Nanomedicine: From where are we coming and where are we going? J Control Release. 2019;311-312:319-321.

15 Lakkireddy HR, Bazile D. Building the design, translation and development principles of polymeric nanomedicines using the case of clinically advanced poly(lactide(glycolide))-poly(ethylene glycol) nanotechnology as a model: An industrial viewpoint. Adv Drug Deliv Rev. 2016;107:289-332.

16 Lakkireddy HR, Bazile DV. Nano-carriers for drug routing - towards a new era. J Drug Target. 2019;27:525541.

17 Alhareth K, Sancey L, Tsapis N, Mignet N. How should we plan the future of nanomedicine for cancer diagnosis and therapy? Int J Pharm. 2017;532(2):657-659. 
18 Hua S, de Matos MBC, Metselaar JM, Storm G. Current Trends and Challenges in the Clinical Translation of Nanoparticulate Nanomedicines: Pathways for Translational Development and Commercialization. Front Pharmacol. 2018;9:790.

19 Wu LP, Wang D, Li Z. Grand challenges in nanomedicine. Mater. Sci. Eng. C. 2020;106:110302.

20 Grainger DW. Connecting drug delivery reality to smart materials design. Int J Pharm. 2013;454:521-4.

21 Boverhof DR, Bramante CM, Butala JH, Clancy SF, Lafranconi M, West J, Gordon SC. Comparative assessment of nanomaterial definitions and safety evaluation considerations. Regul Toxicol Pharmacol. 2015;73:13750.

22 Bremer-Hoffmann S, Halamona-Kensaoui B, Borgos SE. Identifiaction of regulatory needs for nanomedicines. J Interdisciplinary Nanomed. 2018;3:4-15.

23 Gao X, Lowry GV. Progress towards standardized and validated characterizations for measuring physicochemical properties of manufactured nanomaterials relevant to nano health and safety risks. Nanolmpact. 2018; 9:14-30.

24 Halamoda-Kenzaoui B, Holzwarth U, Roebben G, Bogni A, Bremer-Hoffmann S. Mapping of the available standards against the regulatory needs for nanomedicines. Wiley Interdiscip Rev Nanomed Nanobiotechnol. 2019;11:e1531.

25 Caputo F, Clogston J, Calzolai L, Rösslein M, Prina-Mello A. Measuring particle size distribution of nanoparticle enabled medicinal products, the joint view of EUNCL and NCl-NCL. A step by step approach combining orthogonal measurements with increasing complexity. J Control Release. 2019;299:31-43.

26 Caputo F, Arnould A, Bacia M, Ling WL, Rustique E, Texier I, et al. Measuring Particle Size Distribution by Asymmetric Flow Field Flow Fractionation: A Powerful Method for the Preclinical Characterization of LipidBased Nanoparticles. Mol Pharm. 2019;16:756-767.

27 Langevin D, Raspaud E, Mariot S, Knyazev A, Stocco A, Salonen A, et al. Towards reproducible measurement of nanoparticle size using dynamic light scattering: Important controls and considerations. Nanolmpact. 2018;10:161-167.

28 Gioria S, Caputo F, Urbán P, Manus Maguire C, Bremer-Hoffmann S, Prina-Mello A, et al. Are existing standard methods suitable for the evaluation of nanomedicines: some case studies. Nanomedicine. Nanomedicine (Lond). 2018;13:539-554.

29 Jain AK, Thareja S. In-vitro and in-vivo characterization of pharmaceutical nanocarriers used for drug delivery. Artif Cells Nanomed Biotechnol. 2019:47: 524-539.

30 Williams A, Varela E, Meehan E, Tribe K. Characterisation of nanoparticulate systems by hydrodynamic chromatography. Int J Pharm. 2002;242:295-299.

31 Anderson W, Kozak D, Coleman VA, Jämting ÅK, Trau M. A comparative study of submicron particle sizing platforms: accuracy, precision and resolution analysis of polydisperse particle size distributions. $J$ Colloid Interface Sci. 2013;405:322-330.

32 Contado C. Nanomaterials in consumer products: a challenging analytical problem. Front Chem. 2015;3:48.

33 Gross J, Sayle S, Karow AR, Bakowsky U, Garidel P. Nanoparticle tracking analysis of particle size and concentration detection in suspensions of polymer and protein samples: Influence of experimental and data evaluation parameters. Eur J Pharm Biopharm. 2016;104:30-41. doi: 10.1016/j.ejpb.2016.04.013.

34 Kestens, V., Bozatzidis, V., De Temmerman, P., Ramaye Y, Roebben G. Validation of a particle tracking analysis method for the size determination of nano- and microparticles. J Nanopart Res. 2017;19: 271.

35 Danaei M, Dehghankhold M, Ataei S, Hasanzadeh Davarani F, Javanmard R, Dokhani A, et al. Impact of Particle Size and Polydispersity Index on the Clinical Applications of Lipidic Nanocarrier Systems. Pharmaceutics. 2018;10:57.

36 Rasmussen K, Rauscher H, Mech A, Riego Sintes J, Gilliland D, González M, et al. Physico-chemical properties of manufactured nanomaterials - Characterisation and relevant methods. An outlook based on the OECD Testing Programme. Regul Toxicol Pharmacol. 2018;92:8-28.

37 Willmott GR. Tunable Resistive Pulse Sensing: Better Size and Charge Measurements for Submicrometer Colloids. Anal Chem. 2018;90:2987-2995.

38 Lerche D. Comprehensive characterization of nano- and microparticles by In-Situ visualization of particle movement using advanced sedimentation techniques. KONA Powder and Particle Journal 2019;36:156186.

39 Maguire CM, Rösslein M, Wick P, Prina-Mello A. Characterisation of particles in solution - a perspective on light scattering and comparative technologies. Sci Technol Adv Mater. 2018;19:732-745.

40 Kestens, V., Roebben, G., Herrmann, J., Jämting A, Coleman V, Minelli C, et al. Challenges in the size analysis of a silica nanoparticle mixture as candidate certified reference material. J Nanopart Res. 2016; 18:171.

41 Langevin, D.; Lozano, O.; Salvati, A.; Kestens, V.; Monopoli, M.; Raspaud, E.; et al. Inter-Laboratory Comparison of Nanoparticle Size Measurements Using Dynamic Light Scattering and Differential Centrifugal Sedimentation. Nanolmpact 2018; 10;97-107. 
42 Ruseva V, Lyons M, Powell J, Austin J, Malm A, Corbett J. Capillary dynamic light scattering: continuous hydrodynamic particle size from the nano to the micro-scale. Colloids Surf. A Physicochem. Eng. Asp. 2018; 558:505-511.

43 Valero L, Alhareth K, Espinoza Romero J, Viricel W, Leblond J, Chissey A, et al. Liposomes as Gene Delivery Vectors for Human Placental Cells. Molecules. 2018;23(5). pii: E1085.

44 Steinhäusser KG, Sayre PG. Reliability of methods and data for regulatory assessment of nanomaterial risks. Nanolmpact. 2017;7:66-74.

45 ISO 22 412:2008: Particle size analysis - Dynamic light scattering (DLS).

46 ISO 22412:2017: Particle size analysis - Dynamic light scattering (DLS).

47 Lamberty A, Franks K, Braun A, Kestens V, Roebben G, Linsinger TPJ. Interlaboratory comparison for the measurement of particle size and zeta potential of silica nanoparticles in an aqueous suspension. J Nanopart Res. 2011;13:7317-7329.

48 Braun A, Kestens V, Franks K, Roebben G, Lamberty A, Linsinger TPJ. A new certified reference material for size analysis of nanoparticles. J Nanopart Res. 2012;14:1021.

49 Varenne F., Botton J, Merlet C, Beck-Broichsitter M, Legrand FX, Vauthier C. Standardization and validation of a protocol of size measurements by dynamic light scattering for monodispersed stable nanomaterial characterization. Colloids Surf. A Physicochem. Eng. Asp. 2015; 486:124-138.

50 Varenne F, Botton J, Merlet C, Hillaireau H, Legrand FX, Barratt G, et al. Size of monodispersed nanomaterials evaluated by dynamic light scattering: protocol validated for measurements of 60 and 203nm diameter nanomaterials Is now extended to 100 and 400nm. Int J Pharm. 2016;515:245-253.

51 Varenne F, Rustique E, Botton J, Coty JB, Lanusse G, Ait Lahcen M, et al. Towards quality assessed characterization of nanomaterial: transfer of validated protocols for size measurement by dynamic light scattering and evaluation of zeta potential by electrophoretic light scattering. Int J Pharm. 2017; 528:299311.

52 Varenne F, Makky A, Gaucher-Delmas M, Violleau F, Vauthier C. Multimodal dispersion of nanoparticles: A comprehensive evaluation of size distribution with 9 size measurement methods. Pharm Res. 2016;33:1220-1234.

53 Stolnik S., Illum L, Davis S.S. Long circulating microparticulate drug carriers. Adv Drug Deliv Rev. 1995;16:195-214.

54 Alexis F, Pridgen E, Molnar LK, Farokhzad OC. Factors affecting the clearance and biodistribution of polymeric nanoparticles. Mol Pharm. 2008;5:505-515.

55 Jiang W, Kim BY, Rutka JT, Chan WC. Nanoparticle-mediated cellular response is size-dependent. Nat Nanotechnol. 2008;3:145-150.

56 Nel AE, Mädler L, Velegol D, Xia T, Hoek EM, Somasundaran P, et al. Understanding biophysicochemical interactions at the nano-bio interface. Nat Mater. 2009;8:543-557..

57 Liu H, Liu T, Li L, Hao N, Tan L, Meng X, et al. Size dependent cellular uptake, in vivo fate and light-heat conversion efficiency of gold nanoshells on silica nanorattles. Nanoscale. 2012;4:3523-3529.

58 Moghimi MS, Hunter AC, Andresen DL. Factors Controlling Nanoparticle Pharmacokinetics: An Integrated Analysis and Perspective Annu. Rev. Pharmacol. Toxicol. 2012;52:481-503.

59 Kulkarni SA, Feng SS. Effects of particle size and surface modification on cellular uptake and biodistribution of polymeric nanoparticles for drug delivery. Pharm Res. 2013;30:2512-2522.

60 Blanco E, Shen H, Ferrari M. Principles of nanoparticle design for overcoming biological barriers to drug delivery. Nat Biotechnol. 2015;33:941-951.

61 Gustafson HH, Holt-Casper D, Grainger DW, Ghandehari H. Nanoparticle Uptake: The Phagocyte Problem. Nano Today. 2015;10(4):487-510.

62 Hoshyar N, Gray S, Han H, Bao G. The effect of nanoparticle size on in vivo pharmacokinetics and cellular interaction. Nanomedicine (Lond). 2016;11:673-692.

63 Jin Q, Deng Y, Chen X, Ji J. Rational Design of Cancer Nanomedicine for Simultaneous Stealth Surface and Enhanced Cellular Uptake. ACS Nano. 2019;13:954-977.

64 Lundqvist M, Stigler J, Elia G, Lynch I, Cedervall T, Dawson KA. Nanoparticle size and surface properties determine the protein corona with possible implications for biological impacts. PNAS. 2008;105:1426514270.

65 Gaumet M, Vargas A, Gurny R, Delie F. Nanoparticles for drug delivery: the need for precision in reporting particle size parameters. Eur J Pharm Biopharm. 2008;69:1-9.

66 Kang B, Okwieka P, Schöttler S, Seifert O, Kontermann RE, Pfizenmaier K, et al. Tailoring the stealth properties of biocompatible polysaccharide nanocontainers. Biomaterials. 2015;49:125-134.

67 Sun Q, Zhou Z, Qiu N, Shen Y. Rational Design of Cancer Nanomedicine: Nanoproperty Integration and Synchronization. Adv Mater. 2017;29(14): $1606628 .$.

68 Leong HS, Butler KS, Brinker CJ, Azzawi M, Conlan S, Dufés C, et al. On the issue of transparency and reproducibility in nanomedicine. Nat Nanotechnol. 2019;14:629-635. 
69 Rabanel JM, Adibnia V, Tehrani SF, Sanche S, Hildgen P, Banquy X, et al. Nanoparticle heterogeneity: an emerging structural parameter influencing particle fate in biological media? Nanoscale. 2019;11:383-406.

70 Schädlich A, Rose C, Kuntsche J, Caysa H, Mueller T, Göpferich A, et al. How stealthy are PEG-PLA nanoparticles? An NIR in vivo study combined with detailed size measurements. Pharm Res. 2011;28:19952007.

71 Kaasalainen M, Aseyev V, von Haartman E, Karaman DŞ, Mäkilä E, Tenhu H, et al. Size, Stability, and Porosity of Mesoporous Nanoparticles Characterized with Light Scattering. Nanoscale Res Lett. 2017;12:74.

72 Sahin A, Esendagli G, Yerlikaya F, Caban-Toktas S, Yoyen-Ermis D, Horzum U, et al. A small variation in average particle size of PLGA nanoparticles prepared by nanoprecipitation leads to considerable change in nanoparticles' characteristics and efficacy of intracellular delivery. Artif Cells Nanomed Biotechnol. 2017;45:1657-1664.

73 Roda B, Marassi V, Zattoni A, Borghi F, Anand R, Agostoni V, et al. Flow field-flow fractionation and multiangle light scattering as a powerful tool for the characterization and stability evaluation of drug-loaded metal-organic framework nanoparticles. Anal Bioanal Chem. 2018;410:5245-5253.

74 Trindade IC, Pound-Lana G, Pereira DGS, de Oliveira LAM, Andrade MS, Vilela JMC, et al. Mechanisms of interaction of biodegradable polyester nanocapsules with non-phagocytic cells. Eur J Pharm Sci. 2018;124:89-104.

75 Ahmed S, Corvis Y, Gahoual R, Euan A, Lai-Kuen R, Couillaud BM, et al. Conception of nanosized hybrid liposome/poloxamer particles to thicken the interior core of liposomes and delay hydrophilic drug delivery. Int J Pharm. 2019;567:118488.

76 Dobrovolskaia MA, Shurin M, Shvedova AA. Current understanding of interactions between nanoparticles and the immune system. Toxicol Appl Pharmacol. 2016;299:78-89.

77 Szebeni J, Simberg D, González-Fernández Á, Barenholz Y, Dobrovolskaia MA. Roadmap and strategy for overcoming infusion reactions to nanomedicines. Nat Nanotechnol. 2018;13:1100-1108.

78 Passirani C, Barratt G, Devissaguet JP, Labarre D. Long-circulating nanoparticles bearing heparin or dextran covalently bound to poly(methyl methacrylate). Pharm Res. 1998;15:1046-1050.

79 Alhareth K, Vauthier C, Bourasset F, Gueutin C, Ponchel G, Moussa F. Pharmacokinetics and tissue biodistribution in rats of doxorubicin loaded Poly(isobutylcyanoacrylate) nanoparticles prepared by redox radical emulsion polymerization, Eur J Pharm Biopharm. 2012;81(2):453-457.

80 Moghimi SM, Simberg D, Skotland T, Yaghmur A, Hunter AC. The Interplay Between Blood Proteins, Complement, and Macrophages on Nanomedicine Performance and Responses. J Pharmacol Exp Ther. 2019;370:581-592.

81 Simberg D, Moghimi SM. Complement activation by nanomaterials. In: Bonner JC, Brown JM, editors. Interaction of Nanomaterials with the Immune System, Molecular and Integrative Toxicology, Cham Switzerland: Springer Nature 2020. pp 83-98.

82 Neun BW, Ilinskaya AN, Dobrovolskaia MA. Analysis of Complement Activation by Nanoparticles. Methods Mol Biol. 2018;1682:149-160.

83 Vauthier C, Schmidt C, Couvreur P. Measurement of the Density of Polymeric Nanoparticulate Drug Carriers by Isopycnic Centrifugation. J Nanopart Res 1999;1:411-418.

84 Passirani C, Barratt G, Devissaguet JP, Labarre D. Interactions of nanoparticles bearing heparin or dextran covalently bound to poly(methyl methacrylate) with the complement system. Life Sci. 1998;62:775-785.

85 Coty JB, Varenne F, Vachon JJ, Vauthier C. Serial multiple crossed immunoelectrophoresis at a microscale: A stamp-sized 2D immunoanalysis of protein C3 activation caused by nanoparticles. Electrophoresis. 2016;37:2401-2409.

86 Coty JB, Eleamen Oliveira E, Vauthier C. Tuning complement activation and pathway through controlled molecular architecture of dextran chains in nanoparticle corona. Int J Pharm. 2017;532:769-778.

87 Coty JB, Varenne F, Benmalek A, Garsaa O, Le Potier I, Taverna M, et al. Characterization of nanomedicines' surface coverage using molecular probes and capillary electrophoresis. Eur J Pharm Biopharm. 2018;130:4858.

88 Coty JB, Noiray M, Vauthier C. Assessment of Complement Activation by Nanoparticles: Development of a SPR Based Method and Comparison with Current High Throughput Methods. Pharm Res. 2018;35:129.

89 Des Prez RM, Bryan CS, Hawiger J, Colley DG. Function of the classical and alternate pathways of human complement in serum treated with ethylene glycol tetraacetic acid and $\mathrm{MgCl}$-ethylene glycol tetraacetic acid. Infect Immun. 1975;11:1235-1243.

90 Labarre D, Montdargent B, Carreno MP, Maillet F. Strategy for in vitro evaluation of the interactions between biomaterials and complement system. J Appl Biomat. 1993;4:231-240.

91 Hamad I, Al-Hanbali O, Hunter AC, Rutt KJ, Andresen TL, Moghimi SM. Distinct polymer architecture mediates switching of complement activation pathways at the nanosphere-serum interface: implications for stealth nanoparticle engineering. ACS Nano. 2010;4:6629-38. 